\title{
RIdeogram: drawing SVG graphics to visualize and map genome-wide data on the idiograms
}

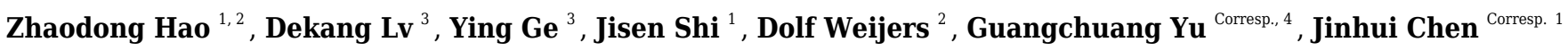 \\ ${ }^{1}$ Key Laboratory of Forest Genetics \& Biotechnology of Ministry of Education, Co-Innovation Center for Sustainable Forestry in Southern China, Nanjing \\ Forestry University, Nanjing, Jiangsu, China \\ 2 Laboratory of Biochemistry, Wageningen University, Wageningen, Haarlem, Netherlands \\ 3 Institute of Cancer Stem Cell, Dalian Medical University, Dalian, Liaoning, China \\ 4 Institute of Bioinformatics, School of Basic Medical Sciences, Southern Medical University, Guangzhou, Guangdong, China \\ Corresponding Authors: Guangchuang Yu, Jinhui Chen \\ Email address: gcyu1@smu.edu.cn, chenjh@njfu.edu.cn
}

Background: Owing to the rapid advances in DNA sequencing technologies, whole genome from more and more species are becoming available at increasing pace. For whole-genome analysis, idiograms provide a very popular, intuitive and effective way to map and visualize the genome-wide information, such as GC content, gene and repeat density, DNA methylation distribution, genomic synteny, etc. However, most available software programs and web servers are available only for a few model species, such as human, mouse and fly, or have limited application scenarios. As more and more non-model species are sequenced with chromosome-level assembly being available, tools that can generate idiograms for a broad range of species and be capable of visualizing more data types are needed to help better understanding fundamental genome characteristics.

Results: The R package Rldeogram allows users to build high-quality idiograms of any species of interest. It can map continuous and discrete genome-wide data on the idiograms and visualize them in a heat map and track labels, respectively.

Conclusion: The visualization of genome-wide data mapping and comparison allow users to quickly establish a clear impression of the chromosomal distribution pattern, thus making RIdeogram a useful tool for any researchers working with omics. 


\section{Rldeogram: drawing SVG graphics to visualize and} 2 map genome-wide data on the idiograms

3

4

Zhaodong $\mathrm{Hao}^{1,2}$, Dekang $\mathrm{Lv}^{3}$, Ying $\mathrm{Ge}^{3}$, Jisen $\mathrm{Shi}^{1}$, Dolf Weijers ${ }^{2}$, Guangchuang $\mathrm{Yu}^{4}$ and Jinhui Chen ${ }^{1}$

${ }^{1}$ Key Laboratory of Forest Genetics \& Biotechnology of Ministry of Education, Co-Innovation Center for Sustainable Forestry in Southern China, Nanjing Forestry University, Nanjing, Jiangsu, China

${ }^{2}$ Laboratory of Biochemistry, Wageningen University, Wageningen, Haarlem, Netherlands

${ }^{3}$ Institute of Cancer Stem Cell, Dalian Medical University, Dalian, Liaoning, China ${ }^{4}$ Institute of Bioinformatics, School of Basic Medical Sciences, Southern Medical University, Guangzhou, Guangdong, China

Corresponding Author:

Guangchuang $\mathrm{Yu}^{4}$ 1023 ShaTaiNan Road, Guangzhou, Guangdong, 510515, China

Email address: gcyu1@smu.edu.cn

Jinhui Chen ${ }^{1}$

159 LongPan Road, Nanjing, Jiangsu, 210037, China

Email address: chenjh@njfu.edu.cn

\section{Abstract}

Background: Owing to the rapid advances in DNA sequencing technologies, whole genome from more and more species are becoming available at increasing pace. For whole-genome analysis, idiograms provide a very popular, intuitive and effective way to map and visualize the genome-wide information, such as GC content, gene and repeat density, DNA methylation distribution, genomic synteny, etc. However, most available software programs and web servers are available only for a few model species, such as human, mouse and fly, or have limited application scenarios. As more and more non-model species are sequenced with chromosomelevel assembly being available, tools that can generate idiograms for a broad range of species and be capable of visualizing more data types are needed to help better understanding fundamental genome characteristics.

Results: The R package RIdeogram allows users to build high-quality idiograms of any species of interest. It can map continuous and discrete genome-wide data on the idiograms and visualize them in a heat map and track labels, respectively. 
38

39

40

41

42

43

44

45

46

47

48

49

50

51

52

53

54

55

56

57

58

59

60

61

62

63

64

65

66

67

68

69

70

71

72

73

74

75

76

77

Conclusion: The visualization of genome-wide data mapping and comparison allow users to quickly establish a clear impression of the chromosomal distribution pattern, thus making RIdeogram a useful tool for any researchers working with omics.

\section{Introduction}

Recently, with the development of sequencing technologies, especially rapid advances in third generation sequencing including Pacific Biosciences (Eid et al. 2009) and Oxford Nanopore Technologies (Laver et al. 2015), BioNano genome mapping (Cao et al. 2014) and Highthroughput chromatin conformation capture sequencing (Dekker et al. 2002), more and more species have their genomes sequenced or updated to the chromosome level (Jiao \& Schneeberger 2017; Phillippy 2017). After the chromosome-level genome completion, an overview of some genome characteristics can help to better understand a species genome, such as gene and transposon distribution across the sunflower genome (Badouin et al. 2017).

An idiogram, also known as a karyotype, is defined as the phenotypic appearance of chromosomes in the nucleus of an eukaryotic cell and has been widely used to visualize the genome-wide data since the first web server, Idiographica, came online in 2007 (Kin \& Ono 2007). There are dozens of tools have been developed for circular genome visualization with a Perl language-based tool Circos being the most used one (Krzywinski et al. 2009; Parveen et al. 2019). In contrast, there are not many alternatives for non-circular plots of whole genome information on idiograms. Although few R packages, like GenomeGraphs (Durinck et al. 2009), ggbio (Yin et al. 2012), IdeoViz (Pai \& Ren 2014), chromPlot (Orostica \& Verdugo 2016) and chromDraw (Janecka \& Lysak 2016), and JavaScript libraries, like Ideogram.js (Weitz et al. 2017) and karyotypeSVG (Prlic 2017), have been developed for non-circular genome visualization, they are either limited in several species and data visualization types or lacking the ample customization. Recently, two R packages, karyoploteR (Gel \& Serra 2017) and chromoMap (Anand 2019), with strengthened capacities have been developed.

However, one function that all these non-circular plots fail to achieve, as Circos does, is to visualize the relationship between two or more species using Bezier curves on idiograms. This function is very useful and allows to interpret genome-wide relationships more intuitively, especially in the visualization of whole genome duplication. Indeed, Circos is usually used to show syntenic blocks both in inter- and intraspecies genome comparisons using Bezier curves (Hu et al. 2019; Wang et al. 2019). Thus, there is a lack of a R package for non-circular genome visualization and allowing to visualize genome-wide relationships between two or more species using Bezier curves on idiograms.

Scalable Vector Graphics (SVG) is a language for describing two-dimensional graphics applications and images. SVG graphics is defined in an eXtensible Markup Language (XML) text file which means that one can easily use any text editor or drawing software to create and edit SVG graphics. Most R graphics packages are built on two graphics systems, the traditional graphics system and the grid graphics system. Here, we developed an R package (RIdeogram) to draw high-quality idiograms without species limitations, that allows to visualize and map whole- 
78

79

80

81

82

83

84

85

86

87

88

89

90

91

92

93

94

95

96

97

98

99

100

101

102

103

104

105

106

107

108

109

110

111

112

113

114

115

116

117

genome information on the idiograms based on the SVG language. Besides, RIdeogram can also be used to show the genome synteny with Bezier curves linking the syntenic blocks on idiograms.

\section{Description}

The package RIdeogram is written in $\mathrm{R}$ ( $\mathrm{R}$ Core Team, 2018), one of the most popular programming languages widely used in statistical computing, data analytics and graphics. However, this new $\mathrm{R}$ graphics package is not built based on any existing graphics systems. We use the $\mathrm{R}$ environment to read the custom input files and calculate the drawing element positions in a coordinate system. Then, we use $\mathrm{R}$ to write all element information into a text file following the XML format which are used to define graphics by the SVG language. A list of the currently implemented commands is given in Table 1. In general, there are three main functions, GFFex, ideogram and convertSVG implemented in the package RIdeogram. Users can use the function data to load the example data or the basic $\mathrm{R}$ function read.table to load the custom data from local files. The function GFFex can be used to extract the information from a GFF3 format genome annotation file. Then, the function ideogram can be used to compute the information for all drawing elements based on the input files and generate a A4-sized SVG file containing a vector graphic which can be conveniently viewed and modified using the software Adobe Illustrator or Inkscape. Alternatively, users can also use the function convertSVG to convert this SVG file into an adjustable image format (pdf, png, tiff, or jpg) with a user-defined resolution according to the practical requirements.

In general, there are two types of data, i.e., continuous and discrete data. For mapping and visualizing, RIdeogram considers the continuous data, such as gene density across the whole genome in $1-\mathrm{Mb}$ windows, as overlaid features and maps them on the idiograms with dark/light colors representing high/low values. For the other data type that are scattered throughout the whole genome, such as the chromosomal distribution of members in one gene family, RIdeogram can add track labels next to the idiograms with three shapes (box, circle and triangle) available to represent different characteristics of these members, such as the subclade that one gene member belongs to. Users can also combine the shapes and colors to represent more than three distinct characteristic types. Furthermore, users can also map the continuous data as a heatmap, a line or area chart along the idiograms. In addition, RIdeogram also provides functions for the visualization of dual and ternary genome synteny using Bezier curves on the idiograms.

RIdeogram is available through CRAN (https://cran.r-project.org/web/packages/RIdeogram/) and is developed on GitHub (https://github.com/TickingClock1992/RIdeogram). Further extensions in development and fixes can be seen in the issue listing page on the package's GitHub page. The new function that we are planning to implement in next version include, but are not limited to, developing more types of data visualization along the idiograms, visualizing genome synteny for more species and enlarging the user-specified genome regions to display detailed characteristics, as we gather more from users. 


\section{Examples}

119 Our first example use the data contained in this package. After the completion of genome

120

121

122

123

124

125

126

127

128

129

130

131

132

133

134

135

136

137

138

139

140

141

142

143

144

145

146

147

148

149

150

151

152

153

154

155

156

157

sequencing, assembly and annotation, RIdeogram can be used to give some idea of how genes are distributed across the whole genome. The example data contained numbers of protein-coding genes calculated in 1-Mb windows which can be considered as continues data and positions of 500 random selected non-coding RNAs, including ribosomal RNAs (rRNAs), transfer RNAs (tRNAs) and microRNAs (miRNAs), which can be considered as discrete data. RIdeogram maps the gene density information on the idiograms as overlaid features in a heat map and adds track labels next to the idiograms with green boxes, purple circles and orange triangles representing rRNAs, tRNAs and miRNAs, respectively (Figure 1). Obviously, inter- and intra-chromosomal gene distributions are non-uniform. For instance, the chromosomal regions adjacent to the centromeres are gene-poor in chromosome 1, 9 and 16 while those are gene-rich in chromosome 11,14 and 17. This function can be applied to many different situations, such as single nucleotide polymorphism (SNP) density and candidate markers (Figure S1 \& Data S1, original data see (Li et al. 2019)), DNA methylation dynamics and potential activated genes (Figure S2 \& Data S2, original data see (Huang et al. 2019)) and transcription factor (TF) binding sites and candidate target genes (Figure S3 \& Data S3, original data see (Shamimuzzaman \& Vodkin 2013)).

Besides visualizing some specific genome characteristics across the whole genome at the chromosome level as showed in Figure 1, RIdeogram can also be used to compare two relevant genome features, such as gene and repeat density, which will provide some important implications for better understanding the relevance of chromosomal distribution patterns of these two features. The example data implemented in this package also contained the information of long terminal repeat (LTR) distribution across the human genome. Since the transposable elements have been suggested to have a potential detrimental effect on gene expression (Hollister \& Gaut 2009), the distributions of gene and LTR are supposed to be opposite across the whole genome as a result of natural selection. As expect, the region that has a relatively high gene content usually has a relatively low LTR density and vice versa (Figure S4), indicating that LTR seems to avoid inserting in the regions with a high gene content in the genome. This similar phenomenon was also observed in the sunflower genome explained using two idiogram graphics, one showing the gene distribution and the other showing the LTR distribution (Badouin et al. 2017). Using RIdeogram, users can integrate these two graphics into one, much easier for researchers to interpret and readers to understand. Apart from the differences, this function can also be used to show the similarities, like the similar genetic diversity patterns across the whole genome between two geographical groups of the same species, in different label types (Figure S5 \& Data S4, Figure S6, original data see (Chen et al. 2019)).

In addition, RIdeogram can also be used to show syntenic comparisons between two or three genomes. As shown in Figure 2, the syntenic blocks between each pair of species, which were identified using MCScan (Tang et al. 2008), were plotted. Particularly, a typical ancestral region in the basal angiosperm Amborella can be tracked to up to two regions in Liriodendron and to up

Peer] Comput. Sci. reviewing PDF | (CS-2019:08:40730:2:0:NEW 3 Dec 2019) 
158

159

160

161

162

163

164

165

166

167

168

169

170

171

172

173

174

175

176

177

178

179

180

181

182

183

184

185

186

187

188

189

190

191

192

193

194

195

196

197

198

199

200

201

to three regions in grape. Based on the fact that no lineage-specific polyploidy event has been found in Amborella and a whole-genome triplication has been detected in grape, it is reasonable to assume a single Liriodendron lineage-specific whole genome duplication event (Chen et al. 2019). Furthermore, RIdeogram allows to visualize a dual genome comparison, such as the genome synteny between human and mouse (Fig. S7 and Data S5). Compared to autosomes, the syntenic blocks between human and mouse $\mathrm{X}$ chromosomes occupy almost the entirety of each $\mathrm{X}$ chromosome, suggesting a highly conserved syntenic relationship of the $\mathrm{X}$ chromosome within the eutherian mammalian lineage (Ross et al. 2005).

\section{Conclusion}

The RIdeogram package provides an efficient and effective way to build idiograms with no species limitations and map genome-wide information on the idiograms for better visualizing and understanding the chromosomal distribution patterns of some particular genomic features.

Meanwhile, this package can be also used to visualize syntenic analysis between genomes. Additionally, it is user-friendly and accessible for biologists without extensive computer programming expertise. Finally, RIdeogram can generate two types of images, a vector graphic or a bitmap file, both in high-quality and meeting conventional requirements for direct use in presentations or journal publications.

\section{Acknowledgements}

We thank Dr. Zhongjuan Zhang for her comments on the manuscript.

\section{References}

Anand L. 2019. chromoMap: Interactive Visualization and Mapping of Chromosomes. bioRxiv. DOI: $10.1101 / 605600$

Badouin H, Gouzy J, Grassa CJ, Murat F, Staton SE, Cottret L, Lelandais-Briere C, Owens GL, Carrere S, Mayjonade B, Legrand L, Gill N, Kane NC, Bowers JE, Hubner S, Bellec A, Berard A, Berges H, Blanchet N, Boniface MC, Brunel D, Catrice O, Chaidir N, Claudel C, Donnadieu C, Faraut T, Fievet G, Helmstetter N, King M, Knapp SJ, Lai Z, Le Paslier MC, Lippi Y, Lorenzon L, Mandel JR, Marage G, Marchand G, Marquand E, BretMestries E, Morien E, Nambeesan S, Nguyen T, Pegot-Espagnet P, Pouilly N, Raftis F, Sallet E, Schiex T, Thomas J, Vandecasteele C, Vares D, Vear F, Vautrin S, Crespi M, Mangin B, Burke JM, Salse J, Munos S, Vincourt P, Rieseberg LH, and Langlade NB. 2017. The sunflower genome provides insights into oil metabolism, flowering and Asterid evolution. Nature 546:148-152. DOI: 10.1038/nature22380

Cao H, Hastie AR, Cao D, Lam ET, Sun Y, Huang H, Liu X, Lin L, Andrews W, Chan S, Huang S, Tong X, Requa M, Anantharaman T, Krogh A, Yang H, Cao H, and Xu X. 2014. Rapid detection of structural variation in a human genome using nanochannel-based genome mapping technology. Gigascience 3:34. DOI: 10.1186/2047-217X-3-34

Chen J, Hao Z, Guang X, Zhao C, Wang P, Xue L, Zhu Q, Yang L, Sheng Y, Zhou Y, Xu H, Xie H, Long X, Zhang J, Wang Z, Shi M, Lu Y, Liu S, Guan L, Zhu Q, Yang L, Ge S, Cheng T, Laux T, Gao Q, Peng Y, Liu N, Yang S, and Shi J. 2019. Liriodendron genome sheds light on angiosperm phylogeny and species-pair differentiation. Nature Plants 5:18-25. DOI: $10.1038 / \mathrm{s} 41477-018-0323-6$ 
202

203

204

205

206

207

208

209

210

211

212

213

214

215

216

217

218

219

220

221

222

223

224

225

226

227

228

229

230

231

232

233

234

235

236

237

238

239

240

241

242

243

244

245

246

247

248

249

250

Dekker J, Rippe K, Dekker M, and Kleckner N. 2002. Capturing chromosome conformation. Science 295:1306-1311. DOI: 10.1126/science.1067799

Durinck S, Bullard J, Spellman PT, and Dudoit S. 2009. GenomeGraphs: integrated genomic data visualization with R. Bmc Bioinformatics 10. DOI: 10.1186/1471-2105-10-2

Eid J, Fehr A, Gray J, Luong K, Lyle J, Otto G, Peluso P, Rank D, Baybayan P, Bettman B, Bibillo A, Bjornson K, Chaudhuri B, Christians F, Cicero R, Clark S, Dalal R, Dewinter A, Dixon J, Foquet M, Gaertner A, Hardenbol P, Heiner C, Hester K, Holden D, Kearns G, Kong X, Kuse R, Lacroix Y, Lin S, Lundquist P, Ma C, Marks P, Maxham M, Murphy D, Park I, Pham T, Phillips M, Roy J, Sebra R, Shen G, Sorenson J, Tomaney A, Travers K, Trulson M, Vieceli J, Wegener J, Wu D, Yang A, Zaccarin D, Zhao P, Zhong F, Korlach $\mathrm{J}$, and Turner S. 2009. Real-time DNA sequencing from single polymerase molecules. Science 323:133-138. DOI: 10.1126/science.1162986

Gel B, and Serra E. 2017. karyoploteR: an R/Bioconductor package to plot customizable genomes displaying arbitrary data. Bioinformatics 33:3088-3090. DOI: 10.1093/bioinformatics/btx346

Hollister JD, and Gaut BS. 2009. Epigenetic silencing of transposable elements: a trade-off between reduced transposition and deleterious effects on neighboring gene expression. Genome Research 19:1419-1428. DOI: 10.1101/gr.091678.109

Hu L, Xu Z, Wang M, Fan R, Yuan D, Wu B, Wu H, Qin X, Yan L, Tan L, Sim S, Li W, Saski CA, Daniell H, Wendel JF, Lindsey K, Zhang X, Hao C, and Jin S. 2019. The chromosomescale reference genome of black pepper provides insight into piperine biosynthesis. Nat Commun 10:4702. DOI: 10.1038/s41467-019-12607-6

Huang H, Liu R, Niu Q, Tang K, Zhang B, Zhang H, Chen K, Zhu JK, and Lang Z. 2019. Global increase in DNA methylation during orange fruit development and ripening. Proceedings of the National Academy of Sciences of the United States of America 116:1430-1436. DOI: $10.1073 /$ pnas.1815441116

Janecka J, and Lysak MA. 2016. chromDraw: an R package for visualization of linear and circular karyotypes. Chromosome Research 24:217-223. DOI: 10.1007/s10577-0159513-5

Jiao WB, and Schneeberger K. 2017. The impact of third generation genomic technologies on plant genome assembly. Current Opinion in Plant Biology 36:64-70. DOI: 10.1016/j.pbi.2017.02.002

Kin T, and Ono Y. 2007. Idiographica: a general-purpose web application to build idiograms ondemand for human, mouse and rat. Bioinformatics 23:2945-2946. DOI: 10.1093/bioinformatics/btm455

Krzywinski M, Schein J, Birol I, Connors J, Gascoyne R, Horsman D, Jones SJ, and Marra MA. 2009. Circos: An information aesthetic for comparative genomics. Genome Research 19:1639-1645. DOI: 10.1101/gr.092759.109

Laver T, Harrison J, O'Neill PA, Moore K, Farbos A, Paszkiewicz K, and Studholme DJ. 2015. Assessing the performance of the Oxford Nanopore Technologies MinION. Biomolecular Detection and Quantification 3:1-8. DOI: 10.1016/j.bdq.2015.02.001

Li X, Singh J, Qin M, Li S, Zhang X, Zhang M, Khan A, Zhang S, and Wu J. 2019. Development of an integrated 200K SNP genotyping array and application for genetic mapping, genome assembly improvement and genome wide association studies in pear (Pyrus). Plant Biotechnology Journal 17:1582-1594. DOI: 10.1111/pbi.13085

Orostica KY, and Verdugo RA. 2016. chromPlot: visualization of genomic data in chromosomal context. Bioinformatics 32:2366-2368. DOI: 10.1093/bioinformatics/btw137

Pai S, and Ren J. 2014. IdeoViz: Plots data (continuous/discrete) along chromosomal ideogram. $R$ package version 1.8.0.

Peer] Comput. Sci. reviewing PDF | (CS-2019:08:40730:2:0:NEW 3 Dec 2019) 
251

252

253

254

255

256

257

258

259

260

261

262

263

264

265

266

267

268

269

270

271

272

273

274

275

276

277

278

279

280

281

282

283

284

285

286

287

288

289

290

291

292

293

294

295

296

297

298

299

300

Parveen A, Khurana S, and Kumar A. 2019. Overview of Genomic Tools for Circular Visualization in the Next-generation Genomic Sequencing Era. Curr Genomics 20:90-99. DOI: $10.2174 / 1389202920666190314092044$

Phillippy AM. 2017. New advances in sequence assembly. Genome Research 27:xi-xiii. DOI: 10.1101/gr.223057.117

Prlic A. 2017. KaryotypeSVG - SVG based ideograms of chromosomes showing cytogenetic bands. 0.2.0 ed.

Ross MT, Grafham DV, Coffey AJ, Scherer S, McLay K, Muzny D, Platzer M, Howell GR, Burrows C, Bird CP, Frankish A, Lovell FL, Howe KL, Ashurst JL, Fulton RS, Sudbrak R, Wen GP, Jones MC, Hurles ME, Andrews TD, Scott CE, Searle S, Ramser J, Whittaker A, Deadman R, Carter NP, Hunt SE, Chen R, Cree A, Gunaratne P, Havlak P, Hodgson A, Metzker ML, Richards S, Scott G, Steffen D, Sodergren E, Wheeler DA, Worley KC, Ainscough R, Ambrose KD, Ansari-Lari MA, Aradhya S, Ashwell RIS, Babbage AK, Bagguley CL, Ballabio A, Banerjee R, Barker GE, Barlow KF, Barrett IP, Bates KN, Beare DM, Beasley H, Beasley O, Beck A, Bethel G, Blechschmidt K, Brady N, BrayAllen S, Bridgeman AM, Brown AJ, Brown MJ, Bonnin D, Bruford EA, Buhay C, Burch P, Burford D, Burgess J, Burrill W, Burton J, Bye JM, Carder C, Carrel L, Chako J, Chapman JC, Chavez D, Chen E, Chen G, Chen Y, Chen ZJ, Chinault C, Ciccodicola A, Clark SY, Clarke G, Clee CM, Clegg S, Clerc-Blankenburg K, Clifford K, Cobley V, Cole CG, Conquer JS, Corby N, Connor RE, David R, Davies J, Davis C, Davis J, Delgado O, DeShazo D, Dhami P, Ding Y, Dinh H, Dodsworth S, Draper H, Dugan-Rocha S, Dunham A, Dunn M, Durbin KJ, Dutta I, Eades T, Ellwood M, Emery-Cohen A, Errington H, Evans KL, Faulkner L, Francis F, Frankland J, Fraser AE, Galgoczy P, Gilbert J, Gill R, Glockner G, Gregory SG, Gribble S, Griffiths C, Grocock R, Gu YH, Gwilliam R, Hamilton C, Hart EA, Hawes A, Heath PD, Heitmann K, Hennig S, Hernandez J, Hinzmann B, Ho S, Hoffs M, Howden PJ, Huckle EJ, Hume J, Hunt PJ, Hunt AR, Isherwood J, Jacob L, Johnson D, Jones S, de Jong PJ, Joseph SS, Keenan S, Kelly S, Kershaw JK, Khan Z, Kioschis P, Klages S, Knights AJ, Kosiura A, Kovar-Smith C, Laird GK, Langford C, Lawlor S, Leversha M, Lewis L, Liu W, Lloyd C, Lloyd DM, Loulseged H, Loveland JE, Lovell JD, Lozado R, Lu J, Lyne R, Ma J, Maheshwari M, Matthews LH, McDowall J, McLaren S, McMurray A, Meidl P, Meitinger T, Milne S, Miner G, Mistry SL, Morgan M, Morris S, Muller I, Mullikin JC, Nguyen N, Nordsiek G, Nyakatura G, O'Dell CN, Okwuonu G, Palmer S, Pandian R, Parker D, Parrish J, Pasternak S, Patel D, Pearce AV, Pearson DM, Pelan SE, Perez L, Porter KM, Ramsey Y, Reichwald K, Rhodes S, Ridler KA, Schlessinger D, Schueler MG, Sehra HK, Shaw-Smith C, Shen H, Sheridan EM, Shownkeen R, Skuce CD, Smith ML, Sotheran EC, Steingruber HE, Steward CA, Storey R, Swann RM, Swarbreck D, Tabor PE, Taudien S, Taylor T, Teague B, Thomas K, Thorpe A, Timms K, Tracey A, Trevanion S, Tromans AC, d'Urso M, Verduzco D, Villasana D, Waldron L, Wall M, Wang QY, Warren J, Warry GL, Wei $\mathrm{XH}$, West A, Whitehead SL, Whiteley MN, Wilkinson JE, Willey DL, Williams G, Williams L, Williamson A, Williamson H, Wilming L, Woodmansey RL, Wray PW, Yen J, Zhang JK, Zhou JL, Zoghbi H, Zorilla S, Buck D, Reinhardt R, Poustka A, Rosenthal A, Lehrach $\mathrm{H}$, Meindl A, Minx PJ, Hillier LW, Willard HF, Wilson RK, Waterston RH, Rice CM, Vaudin M, Coulson A, Nelson DL, Weinstock G, Sulston JE, Durbin R, Hubbard T, Gibbs RA, Beck S, Rogers J, and Bentley DR. 2005. The DNA sequence of the human X chromosome. Nature 434:325-337. DOI: 10.1038/nature03440

Shamimuzzaman M, and Vodkin L. 2013. Genome-wide identification of binding sites for NAC and YABBY transcription factors and co-regulated genes during soybean seedling development by ChIP-Seq and RNA-Seq. BMC Genomics 14:477. DOI: 10.1186/14712164-14-477

Peer] Comput. Sci. reviewing PDF | (CS-2019:08:40730:2:0:NEW 3 Dec 2019) 
301

302

303

304

305

306

307

308

309

310

311

312

313

314
Tang HB, Wang XY, Bowers JE, Ming R, Alam M, and Paterson AH. 2008. Unraveling ancient hexaploidy through multiply-aligned angiosperm gene maps. Genome Research 18:1944-1954. DOI: 10.1101/gr.080978.108

Wang M, Tu L, Yuan D, Zhu, Shen C, Li J, Liu F, Pei L, Wang P, Zhao G, Ye Z, Huang H, Yan F, Ma Y, Zhang L, Liu M, You J, Yang Y, Liu Z, Huang F, Li B, Qiu P, Zhang Q, Zhu L, Jin S, Yang X, Min L, Li G, Chen LL, Zheng H, Lindsey K, Lin Z, Udall JA, and Zhang X. 2019. Reference genome sequences of two cultivated allotetraploid cottons, Gossypium hirsutum and Gossypium barbadense. Nat Genet 51:224-229. DOI: 10.1038/s41588018-0282-x

Weitz EM, Pantano L, Zhu J, Upton B, and Busby B. 2017. Viewing RNA-seq data on the entire human genome. F1000Res 6:596. DOI: 10.12688/f1000research.9762.1

Yin TF, Cook D, and Lawrence M. 2012. ggbio: an R package for extending the grammar of graphics for genomic data. Genome Biology 13. DOI: 10.1186/gb-2012-13-8-r77 


\section{Table $\mathbf{1}$ (on next page)}

Table 1. Functions contained in the package R/deogram. 
1 Table 1. Functions contained in the package RIdeogram.

\begin{tabular}{ll}
\hline Function name & Description \\
\hline GFFex & Extract information from a GFF3 format genome annotation fill. \\
ideogram & Map and visualize the genome-wide data on the idiograms \\
convertSVG & Convert the output file from the SVG format to the format users chose. \\
svg2tiff & Convert the output file from the SVG format to the TIFF format. \\
svg2pdf & Convert the output file from the SVG format to the PDF format. \\
svg2jpg & Convert the output file from the SVG format to the JPG format. \\
svg2png & Convert the output file from the SVG format to the PNG format. \\
\hline
\end{tabular}

2 


\section{Figure 1}

Figure 1. Gene distribution across the whole human genome.

The overlaid heatmap shows the gene density and the tack labels refer to 500 random selected RNAs consisted of rRNAs (green boxes), tRNA (purple circles) and miRNA (orange triangles) locus across the human genome. Annotation information was downloaded from the GENCODE website (https://www.gencodegenes.org). 


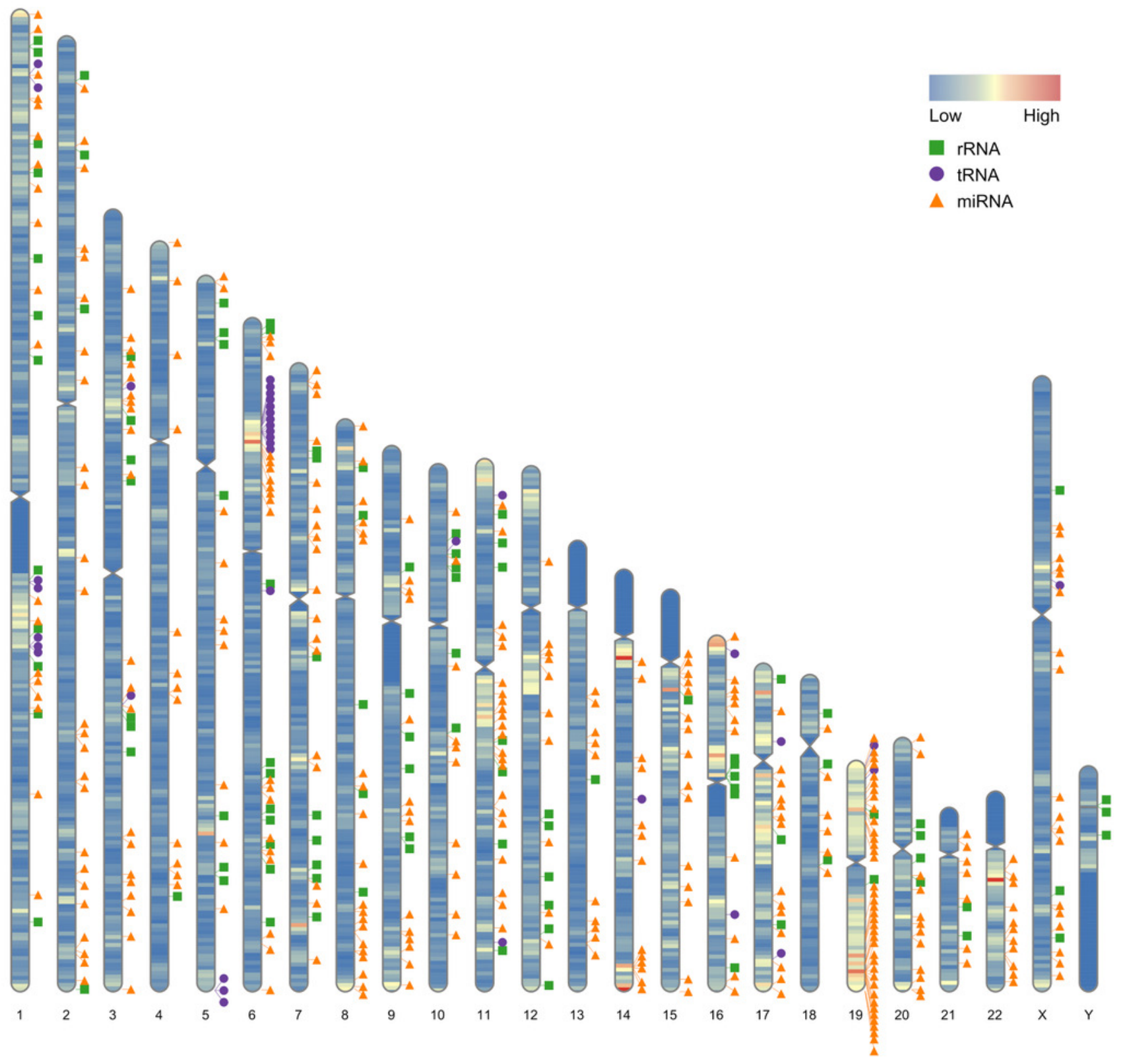


Figure 2

Figure 2. Syntenic comparison of three plant genomes.

Genome synteny patterns show that a typical ancestral region in the basal angiosperm Amborella can be tracked to up to two regions in Liriodendron and to up to three regions in grape. Gray wedges in the background highlight major syntenic blocks spanning more than 30 genes between the genomes (highlighted by one syntenic set shown in colored).

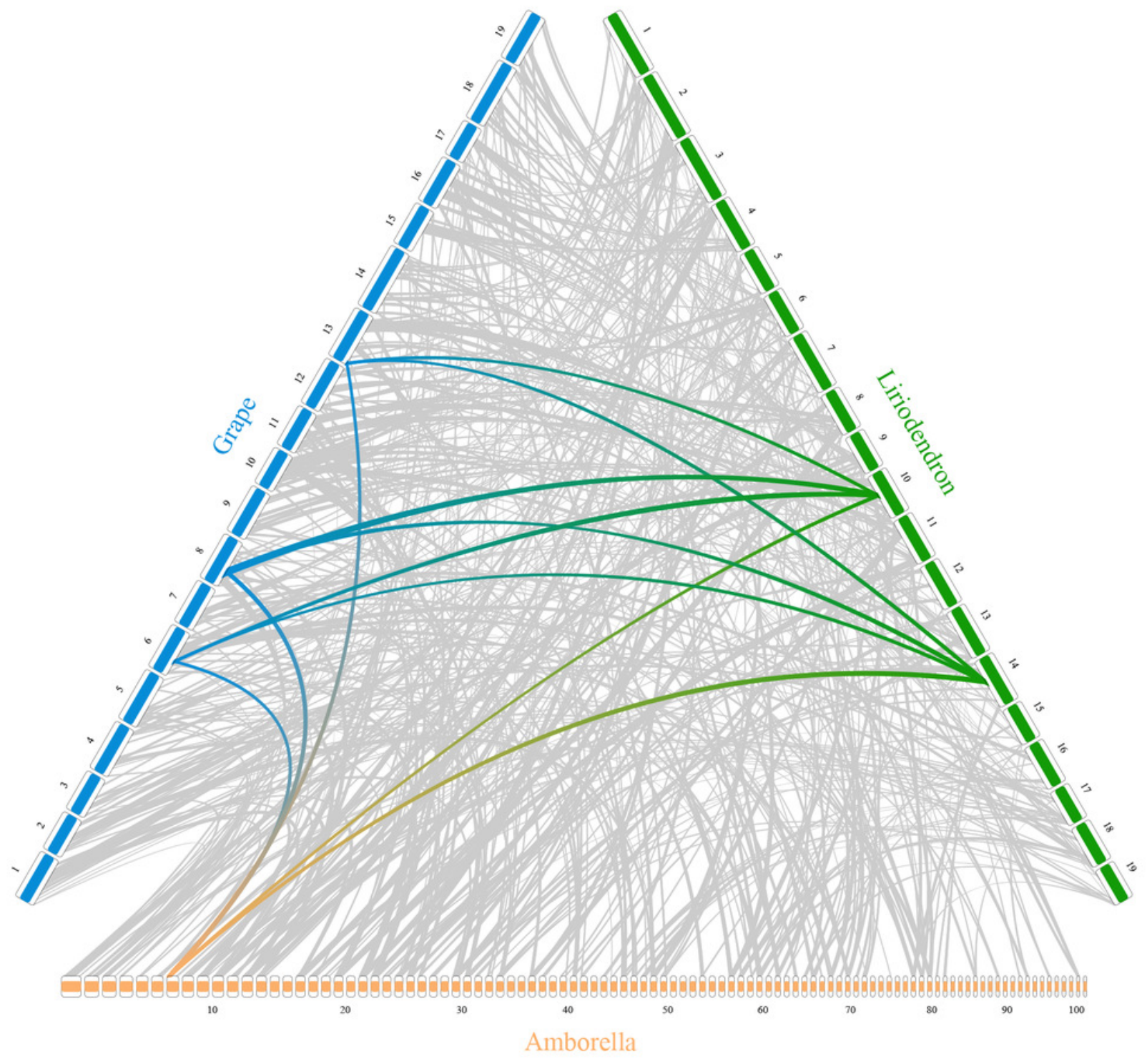

